\title{
PRÉCIS OF UNCUT
}

\section{Précis de Uncut}

\author{
DAVID RIPLEY ${ }^{a}$ \\ davewripley@gmail.com \\ ${ }^{a}$ Philosophy Department, Monash University, Australia.
}

\begin{abstract}
Uncut is a book about two kinds of paradoxes: paradoxes involving truth and its relatives, like the liar paradox, and paradoxes involving vagueness. There are lots of ways to look at these paradoxes, and lots of puzzles generated by them, and Uncut ignores most of this variety to focus on a single issue. That issue: do our words mean what they seem to mean, and if so, how can this be? I claim that our words do mean what they seem to, and yet our language is not undermined by paradox. By developing a distinctive theory of meaning, I show how this can be.
\end{abstract}

Key words: Paradox; Truth; Vagueness; Validity; Meaning.

\section{Resumen}

Uncut es un libro sobre dos tipos de paradojas: paradojas que involucran la noción verdad y otros conceptos similares, como la paradoja del mentiroso, y las paradojas de la vaguedad. Hay muchas formas de entender estas paradojas y son muchos los enigmas que estas generan. Uncut ignora gran parte de esto para concentrarse en un único asunto: ¿tienen nuestras palabras el significado que aparentan tener? Y si es así, ¿cómo es esto posible? Sugiero que nuestras palabras tienen el significado que aparentan tener y sin embargo nuestro lenguaje no se ve afectado por las paradojas. Para mostrar cómo esto es posible, desarrollo una teoría distintiva del significado.

Palabras clave: Paradojas; Verdad; Vaguedad; Validez; Significado.

\section{Introduction}

Uncut is a book about two kinds of paradoxes: paradoxes involving truth and its relatives, like the liar paradox, and paradoxes involving vagueness. There are lots of ways to look at these paradoxes, and lots of puzzles generated by them, and Uncut ignores most of this variety to focus on a single question. That question: do our words mean what they seem to mean, and if so, how can this be? 
Consider the liar paradox: using just a few basic principles involving truth and negation, we can generate a proof of any sentence you like from the mere existence of a sentence like 'This very sentence is not true'. (We'll look at this in more detail later in this piece.) Since sentences like that one clearly do exist, it's tempting to think that some of those basic principles about truth and negation can't really hold. The trouble is that these principles are so basic that they've often been taken to be necessary for 'true' and 'not' to mean what they seem to mean. The issue with vagueness is parallel: again, we have arguments leading to unacceptable conclusions that work by way of principles seemingly guaranteed by the meanings of vague words, and maybe also by the meanings of words like 'if'.

To accept that these words mean what they seem to mean, then, naturally goes with a certain defeatism in the face of the paradoxes. If all the principles in the paradoxical arguments really are correct, then it's seemed to many that those arguments must really go through, and so their conclusions are established. But this must be mistaken. These conclusions-which are arbitrary or nearly so (depending on which paradoxes we're talking about)—are not in fact established by the paradoxical arguments. Not everything is true, Chewbacca is not bald, and what remains is to figure out how on Earth this can possibly be so in light of the paradoxes.

Along these lines, it's natural to think that the error must be in one or more of the principles involved in the reasoning: perhaps some of 'true', 'not', and so on don't really mean what they seem to mean, or maybe their meaning what they seem to mean doesn't really require all the basic principles to hold that are used in the paradoxical arguments.

Here there's a choice point in the literature on these paradoxes: some people think that the principles involving truth are non-negotiable, and so adopt a non-obvious theory of negation, while other people think that the principles involving negation are non-negotiable, and so adopt a non-obvious theory of truth. Similar choices arise in discussions of other paradoxes. This is the distinction between 'classical' and 'nonclassical' theories of these various paradoxes: 'classical' theories adopt nonobvious theories of truth, vague predicates, and so on; while 'nonclassical' theories adopt non-obvious theories of 'not', 'if', and so on. There's lots of interesting material that's been developed in service of both kinds of approach. But either way you go, you end up ruling out a certain package deal: the package that says that truth and negation really do mean just what they appear to mean, and that this really does suffice for them to obey the basic principles involved in the paradoxical argument. 
To hang on to this package, we need to locate the trouble elsewhere. Where Uncut locates the trouble is not in the basic principles themselves, but in the way they get put together in the paradoxical arguments. (This is, very roughly, what makes an approach to paradoxes substructural; see Ripley (2015b) and Shapiro (2016).) To think carefully about combining principles in this way, it helps to have a background theory of meaning in place, to see just what kinds of combination do and don't make sense. If we adopt the right kind of theory of meaning at the outset, it turns out that it's clear where to locate the trouble. There's one particular way of combining different principles that 1) turns up in every paradoxical argument, and 2) cannot be justified. This is the mode of combination known to proof theorists as cut.

The plan of the book, and of this precis, is to start by presenting this kind of theory of meaning, and then go on to add in the needed principles connected to various pieces of vocabulary. Along the way, I'll pause a few times to show (or here in the précis, often simply to claim) that no trouble has resulted: the problematic conclusions simply do not follow.

\section{Thinking about Meaning}

I'll open by giving a way of thinking about linguistic meaning that turns on the notions of positions and bounds. The general idea I'm taking up here is due to Greg Restall, who's developed it in a number of places, including Restall $(2005,2009,2013,2008)$. I won't argue for it here (and I don't argue for it in the book), except to note that it makes possible the kind of solution to the paradoxes I'm after. ${ }^{1}$

\section{Positions and bounds}

The approach Uncut takes to meaning is centered on the speech acts of assertion and denial, and certain norms that govern collections of these acts. I'll refer to a collection of assertions and denials as a position, and talk of positions as being in bounds or out of bounds.

These bounds are understood as a social kind: they are created and sustained by the place they occupy in our practices. As is usual for

\footnotetext{
${ }^{1}$ I begin to defend this way of thinking about meaning more directly in Ripley (2017a). Restall has argued (in each of the above-cited pieces of his) that this approach does require cut, and so does not make possible the kind of solution Uncut offers. I respond to those arguments in Ripley (2013a, 2015a), and Uncut itself, but here I'll just skip over the issue.
} 
social kinds, understanding what it is for a position to be in or out of bounds needs to start from understanding what it is to treat a position as in or out of bounds. (Compare: to explain what it is to be royalty, start from what it is to treat someone as royalty.) Uncut and Ripley (2017a) go into more depth about this; here I'll just sketch the rough idea. Some positions simply don't fit together. For example, a position that asserts and denies the very same thing doesn't add up. A position that asserts 'Melbourne is bigger than Canberra' and 'Canberra is bigger than Darwin' while denying 'Melbourne is bigger than Darwin' also doesn't add up.

If someone seems to have adopted a position like this, we might look for some other way to interpret what they've said, so that they didn't really adopt the position. (Maybe they meant 'bigger' in terms of population size in some of their utterances, and in terms of font size on a particular map in others.) In the course of looking for such an interpretation, we might ask them for clarification ('A minute ago I thought you said $x$. But now you seem to be saying $y$. What's up?'). Or, rather than look for an interpretation that works, we might simply dismiss them as talking crap, as not a serious interlocutor. All of these kinds of responses-reinterpretation, asking for clarification, dismissal-are enforcement behaviours important to this social kind. Some positions are taken seriously as the kind of thing someone might mean; others are not. This taking seriously or not is the key activity we engage in to establish the bounds. ${ }^{2}$

\section{The bare calculus}

Those, anyway, are the core ideas I want to work with. It helps to be able to investigate these ideas precisely and carefully, though, if we can prove things about them. So I want to formalize this family of ideas to be able to see how it all comes together.

To this end, Uncut uses a usual first-order language with equality. This first-order formal language is to be understood as a clumsy model of the languages we actually speak. In particular, then, some of its

\footnotetext{
${ }^{2}$ Which positions we treat as in bounds-and maybe even which positions are in bounds-varies from context to context. For example, in most normal contexts, a position that asserts both 'Napoleon died in 1815' and 'Napoleon fought at Waterloo in 1821' is out of bounds. But in a conversational context where the participants are taking seriously the possibility of resurrection or zombie soldiers, perhaps it is not. The extent to which the bounds vary with context is the extent to which meanings vary with context. Fwiw, my sympathies here lie with reasonably strong forms of contextualism; but that's an issue for another day.
} 
predicates are vague, one of its predicates is a truth predicate, some of its names are names for its own sentences, and so on. Of course, these statuses are not syntactic statuses, so nothing about the syntax of the language will reveal which predicates and names these are. But they are assumed to be present from the get-go. I will give a series of gradually fuller (but always incomplete) theories of this single language. ${ }^{3}$

As above, I understand positions to be collections of assertions and denials. I'll model a position as a pair of sets of sentences, written [ $\Gamma \Rightarrow \Delta]$, where $\Gamma$ and $\Delta$ are sets of sentences. In the position $[\Gamma \Rightarrow \Delta], \Gamma$ should be understood as the collection of sentences asserted, and $\Delta$ as the collection of sentences denied.

It's no accident that this notation for positions resembles a notation used for sequent-style proof theories. The plan is to lay out a theory of which positions are out of bounds, and connections between positions that are out of bounds, and this theory is going to end up looking a lot like sequent-style proof theories. I'll start from a collection of initial positions, taken to be out of bounds, and add rules that allow new positions to be derived from old. The intention is that each position derivable in this way will be out of bounds.

The resemblance to usual sequent-style proof systems is really handy, but possibly misleading. It's handy because it allows me to take advantage of great ideas and results in proof theory in a completely straightforward way. Formally, what I'm doing just is constructing a particular proof system rule by rule, and it's very nice to not have to reinvent the wheel.

At the same time, though, the similarity to familiar proof systems might be misleading. This is because it might suggest that my project is more intimately involved in debates about logic than it really is. But Uncut makes no claims at all about logic: not about logical consequence, not about logical vocabulary, not about the normative status of logic, not about continuity (or lack thereof) with the natural sciences. I am not offering any theory of 'the logic of the paradoxes'. My topic is meaning in natural language, and how to understand it in light of the paradoxes. In this work logic is a tactic, not a topic.

But then we can't just assume that ordinary logicky rules transfer straightforwardly to become plausible claims about bounds on positions. Even if such rules are correct when interpreted as about logic, that says

\footnotetext{
${ }^{3}$ And not! a series of complete theories of gradually fuller (but always incomplete) fragments. The difference is mainly one of style, but it's key to be clear about which style is in play.
} 
nothing about whether they are correct when interpreted as about the bounds. The bounds have to be inspected in their own right.

So I begin with a calculus for the bounds I call the bare calculus, or BC. This calculus contains just two rules, Id and D, explained below. In fact, I think that BC exhausts what can be said about the bounds in general, without attention to any particular vocabulary. But this exhaustion claim is controversial, so let's delay it for now, to get $\mathbf{B C}$ on the table.

The rule Id has it that for any sentence $A$, the position $[A \Rightarrow A]$ is out of bounds. That is, the position that both asserts $A$ and denies $A$ is ruled out. While I offer no theory here about the nature or telos of assertion and denial, this would seem to be a plausible upshot of at least many such theories: whatever assertion and denial are, or are for, they are opposed to each other. ${ }^{4}$

The rule $\mathrm{D}$ (for 'dilution') has it that whenever a position $[\Gamma \Rightarrow \Delta]$ is out of bounds, then any superposition $\left[\Gamma, \Gamma^{\prime} \Rightarrow \Delta, \Delta^{\prime}\right]$ of that position is also out of bounds. ${ }^{5}$ That is: if you're in a hole, and you keep digging, you're still in a hole. If adopting the position $[\Gamma \Rightarrow \Delta]$ violates the bounds, then in order to come back in bounds a speaker must take something back. Keeping on asserting and denying more things won't do it. This is a key place where the project of Uncut diverges from that of Brandom (1994).

These rules can be found in a more usual-looking form in Figure 1 , which gives the bare calculus in schematic fraction-bar form.

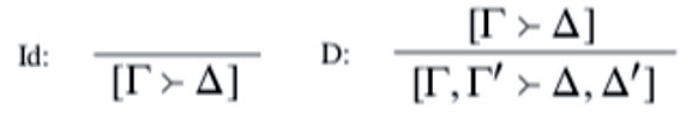

Figure 1: The Bare calculus BC

Admissible \& derivable rules

As a proof system, BC is not terribly interesting. But it is already enough to develop a key distinction: the distinction between admissible rules and derivable rules. A rule is admissible in a calculus iff each instance of the rule meets the following condition: if all the premises of the instance are derivable in the calculus, then the conclusion of that instance is also derivable in the calculus.

Figure 2 contains two examples of rules that are admissible in

\footnotetext{
${ }^{4}$ For one idea about how to get to this constraint from the telos of denial, see Price (1990, sec. 3).

5 'Superposition' here is like 'superset'; there's nothing quantum afoot.
} 
BC: DId (diluted identity) and Swap. To see that they are admissible, start by noting that a position $[\Gamma \Rightarrow \Delta]$ is derivable in BC iff $\Gamma \cap \Delta \neq \emptyset$; BC derives all and only the positions whose assertions and denials overlap. But the conclusion of DId always meets this condition; and if the premise of an instance of Swap meets this condition then so does the conclusion. So both rules are admissible in BC.

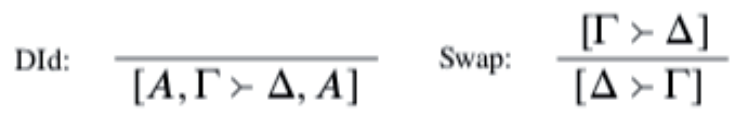

Figure 2: DId and Swap

For a rule to be derivable in a calculus, each instance of the rule must meet a more stringent condition: that if all the premises of the instance are added to the calculus as initial positions, then the conclusion of the instance can be derived in this expanded calculus. DId is not only admissible in BC, but also derivable. (Indeed, any zeropremise rule like DId is admissible iff it is derivable.) But Swap, while admissible, is not derivable in BC. For example, there is an instance of Swap that moves from $[p \Rightarrow q]$ to $[q \Rightarrow p]$. But if we add $[p \Rightarrow q]$ to $\mathbf{B C}$ as a new initial position, there is no derivation in the resulting calculus of $[q \Rightarrow p]$. Rules like this that are admissible but not derivable for a calculus are called merely admissible.

When we add more rules to a calculus, we can sometimes make (merely) admissible rules become inadmissible. We saw an example of this already, in seeing that Swap is not derivable in BC. But in fact, this can happen to any rule $R$ that is merely admissible in a calculus: there is always some way to add rules to the calculus to reach a new calculus for which $R$ is not admissible at all. This can never happen, though with derivable rules: adding to a calculus will never make a derivable rule underivable. This is because derivability, in its definition, already took potential additions into account.

Now, the proof system I am building towards in Uncut is nothing like a complete theory of the bounds. That would be far too much to ask; it would be a complete theory of the meaning of every word in our language. I have no commitment to such a thing being possible even in principle; I certainly am not about to try it. ${ }^{6}$ The proof systems I give

\footnotetext{
${ }^{6}$ For the cognoscenti: yes, I'm disavowing any commitment to 'semantic closure'. Semantic closure is often taken to matter because it figures as a premise in an argument whose conclusion is that English (for example) contains its own truth predicate. (And since it contains its own truth predicate, we need to worry about the
} 
are partial theories of the bounds. If a rule is derivable in the calculi I endorse, then it will remain derivable no matter how these calculi are extended; I am surely committed to the bounds being closed under any such rule. But-and this is why the distinction matters-the same is not true for merely admissible rules. A rule might be merely admissible in one, or even all, of the calculi I endorse, without this carrying any commitment on my part to the bounds being closed under that rule. Its mere admissibility might just be a result of the incompleteness of my theory: there might be counterexamples that my theory is not rich enough to show to be counterexamples.

Indeed, this is just the status that Swap has wrt BC. BC does not have the resources to identify any counterexamples to Swap; and the bounds really do (so I claim) obey all the rules of BC. But nonetheless, the bounds do not obey Swap. It is just that the counterexamples to Swap all turn on the behaviour of particular vocabulary, vocabulary that BC takes no account of.

Cut

Time to look at the rule of cut. I'll work with the rule in this form: ${ }^{7}$

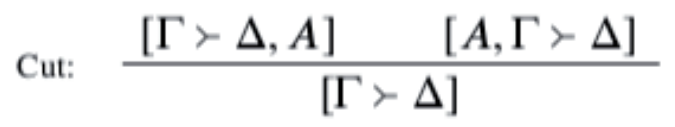

Cut's status in BC is just the same as Swap's: it is merely admissible. So everything I've said so far is compatible with cut's having

liar, etc.) But that English contains its own truth predicate is way more obvious than semantic closure is. I'm happy simply to take it for granted here.

Plus, there's at least some reason to suspect that semantic closure might be wrong. It seems entirely possible for us to adopt social norms that we cannot express in words. Imagine giving a complete story of the norms that constitute politeness! (For example, think about the ways in which two facial expressions can differ such that one of them is polite while the other is not.) And yet etiquette manuals exist, use words to do their best, and are capable of capturing at least some important aspects of the norms at play.

I see no reason why the particular norms that constitute the meanings of our words couldn't be of this type. So while I am indeed offering, in words, a partial theory of these norms, this comes with no commitment to the completability of the theory, even in principle.

${ }^{7}$ Cut comes in many forms, but for present purposes the differences turn out basically not to matter, which is handy. For discussion of some of the variety in the area, see Ripley (2015b, 2017b). 
counterexamples that turn on the behaviour of particular vocabulary, just like Swap does. Indeed, I think this is exactly the case. The only difference is that for Swap, we can already find such vocabulary among the UFO vocabulary, whereas for cut we must look outside.

But before we get to that vocabulary, it's worth a look at cut directly in its own right, to see what constraints on the bounds it encodes. After all, Id and D follow directly from the picture in play of what the bounds are and how they work; if cut were to follow from this picture as well, then my claim that $\mathbf{B C}$ exhausts the vocabulary-independent constraints on the bounds would fail, and there would be no room for any counterexamples.

So what does cut say, in terms of the bounds? It says that if the positions $[\Gamma \Rightarrow \Delta, A]$ and $[A, \Gamma \Rightarrow \Delta]$ are both out of bounds, then we can forget all about $A:[\Gamma \Rightarrow \Delta]$ is out of bounds on its own. That is, if a position $[\Gamma \Rightarrow \Delta]$ rules out in-bounds asserting some claim $A$, and it also rules out in-bounds denying that same $A$, then the position itself is already out of bounds, even if it doesn't say anything about $A$ at all. Contrapositively, we can see cut as an extensibility claim: if a position $[\Gamma \Rightarrow \Delta]$ is in bounds, then one of $[\Gamma \Rightarrow \Delta, A]$ and $[A, \Gamma \Rightarrow \Delta]$ must be too: there must be some in-bounds way to extend [ $\Gamma \Rightarrow \Delta]$ with $A$, either by denying it or by asserting it.

At this point, we have no particular reason to think that this doesn't hold, but we also have no particular reason to think that it does. Why couldn't it be the case that taking up some position $[\Gamma \Rightarrow \Delta]$ rules out taking any stand on $A$, and yet $[\Gamma \Rightarrow \Delta]$ is in bounds? Perhaps the bounds simply require us not to take any stand on $A$. I'm not yet claiming there are such examples, although I will be soon. Rather, I'm claiming that we have no reason to rule out such examples in advance. Whether this kind of extensibility constraint holds is a matter of the global organization of the bounds; it remains to be seen how that shakes out.

\section{Particular Vocabulary}

It might seem like I've said surprisingly little so far about vagueness or truth, for a book about paradoxes. But in fact the trick is already pulled. From here all that remains is to say the most straightforward possible things about the UFO vocabulary, vague predicates, and semantic vocabulary; and to verify that no trouble results. 


\section{UFO Vocabulary}

Meanings of particular pieces of vocabulary are to be given as conditions on the in-bounds assertibility and deniability of sentences involving that vocabulary. Before we get to the particularly paradoxical vocabulary, I'll start by giving meanings for the UFO vocabulary. This helps in two ways: it gives an example of how this kind of approach to meanings works, and it also allows us to examine interactions between paradox-prone vocabulary on the one hand and the UFO vocabulary on the other.

Here, I'll just give meanings for negation $\neg$, conjunction $\wedge$, and the universal quantifier $\forall$. Other UFO vocabulary (except for equality) can be understood as defined from this stock in the familiar way. ${ }^{8}$

Negation, I claim, is for swapping the roles of assertion and denial. Asserting $\neg A$ amounts to the same, as far as the bounds are concerned, as denying $A$; and denying $\neg A$ amounts to the same, as far as the bounds are concerned, as asserting $A$. This is not an uncontentious theory of negation, of course. But it is a familiar, classically-flavoured one. If there is anything surprising or unfamiliar about it, it is the bounds-based way of expressing this idea about negation, not the idea itself.

This idea finds expression in a pair of double-line rules:

$$
\neg \mathrm{L}: \frac{[\Gamma \succ \Delta, A]}{[\neg A, \Gamma \succ \Delta]} \quad \neg \mathrm{R}: \frac{[A, \Gamma \succ \Delta]}{=[\Gamma \succ \Delta, \neg A]}
$$

The double line should be understood as an 'if and only if'; in each rule, the position above the line is out of bounds iff the position below the line is as well. These rules merely encode in symbolic form the theory of negation's meaning floated above: $\neg \mathrm{L}$ tells us when $\neg A$ is assertible (namely, iff $A$ is deniable); and $\neg \mathrm{R}$ tells us when $\neg A$ is deniable (namely, iff $A$ is assertible).

On to conjunction. Asserting a conjunction $A \wedge B$ amounts to the same, as far as the bounds are concerned, as asserting both $A$ and $B$ : the single act is in bounds iff the pair of acts is. And denying a conjunction $A \wedge B$ is out of bounds iff denying $A$ and denying $B$ are both out of bounds. (A conjunction is undeniable iff both conjuncts are.) This is not an uncontentious theory of conjunction's meaning (although conjunction

\footnotetext{
${ }^{8}$ This definitional approach, and the omission of equality, is just to save space here. Uncut presents meanings for each piece of UFO vocabulary directly, and includes equality.
} 
in general is less contentious than negation), but it is again a completely standard one, apart perhaps from the bounds-based idiom in which it is expressed.

This theory finds expression in another pair of double-line rules:

$$
\wedge \mathrm{L}: \frac{[A, B, \Gamma \succ \Delta]}{[[A \wedge B, \Gamma \succ \Delta]} \quad \wedge \mathrm{R}: \frac{[\Gamma \succ \Delta, A] \quad[\Gamma \succ \Delta, B]}{\frac{[\Gamma \succ \Delta, A \wedge B]}{[\Gamma \succ \Delta]}}
$$

The double line again should be read as 'if and only if'. $\wedge R$ is a two-premise double-line rule: this should be read as allowing the bottom position to be derived from the pair of top positions, and as allowing either top position to be derived freely from the bottom position. It thus amounts to 'both premises iff the conclusion', which is what's needed.

The universal quantifier is slightly different from negation and conjunction, in the following way: I'm not here going to give you a full 'if and only if' statement of its assertibility conditions. Rather, I'm going to say things I take to be true about the meaning of the universal quantifier, without claiming to have thereby given an exhaustive theory of its meaning. This is, quite frankly, because I don't know how to give an exhaustive theory of its meaning. Fortunately, though, none is required for my project. At the very least, the following holds: if it is out of bounds to assert $A(t)$ for any term $(t)$, then it must be out of bounds to assert $\forall x A(x)$. Deniability conditions can be pinned down more fully: $\forall x A(x)$ is out of bounds to deny iff $A(y)$ is out of bounds to deny for some variable $a$ in a position that does not otherwise mention $a$. (This idea makes the most sense with one extra rule, to make sure that variables behave like variables; that's on its way.)

This leads us to the following rules:

$$
\forall \mathrm{L}: \frac{[A(t), \Gamma \succ \Delta]}{[\forall x A(x), \Gamma \succ \Delta]} \quad \forall \mathrm{R}: \quad \frac{[\Gamma \succ \Delta, A(a)]}{\overline{[\Gamma \succ \Delta, \forall x A(x)]}}
$$

Note that $\forall \mathrm{L}$ is only a single-line rule; this fits with the one-way conditional claim about the assertion conditions of $\forall$ sentences. ${ }^{9}$ The

${ }^{9}$ If there were some reason to want double-line rules in particular, there is a usual candidate for $\forall \mathrm{L}$ :

$$
\forall L^{\star}: \frac{[A(t), \forall x A(x), \Gamma \succ \Delta]}{[\forall x A(x), \Gamma \succ \Delta]}
$$

But the bottom-to-top direction of this rule is simply an instance of $\mathrm{D}$, so would add nothing to the present proof system. More importantly, it tells us nothing about 
rule $\forall \mathrm{R}$ should be understood as having a side condition: it may only be applied when the variable $a$ does not occur free in $\Gamma \cup \Delta$. If the position $[\Gamma \Rightarrow \Delta]$ already says things about $a$, then ruling out denial of $A(a)$ should not suffice for ruling out denial of $\forall x A(x)$; the denial of $A(a)$ might be ruled for for reasons that have to do with $a$ specifically. But if $[\Gamma \Rightarrow \Delta]$ says nothing at all about $a$, and still manages to make $A(a)$ undeniable, then this must be for reasons that have nothing at all to do with $a$; the position must also make $\forall x A(x)$ undeniable.

In order to state the deniability conditions for $A$, I made use of the notion of a variable. The key idea for variables is that they are mere placeholders, with no special assumptions made about them. So if a position involving a variable is out of bounds, then the corresponding position using any term in place of the variable must be out of bounds as well. ${ }^{10}$ That is, we have the rule VSub:

$$
\text { vSub: } \frac{[\Gamma(a) \succ \Delta(a)]}{[\Gamma(t) \succ \Delta(t)]}
$$

In this rule, $a$ should be understood as a variable with all of its free occurrences indicated, and $t$ is an arbitrary term. As a result, there will be no free $a \mathrm{~s}$ left in the conclusion position. Sequent systems are often designed so that VSub is merely admissible, but recall that I'm trying to avoid appeal to merely admissible rules. To be sure that variables behave like mere placeholders, then, even after new rules are added later, we may as well impose VSub directly.

That, then, gives such a theory as I have to offer here of the meanings of the UFO vocabulary. When the rules from this section are added to BC, we arrive at a calculus I'll call CL. This calculus is sound and complete for first-order classical logic without equality. ${ }^{11}$

That is, a position is derivable in iff it is classically valid. The 'if' direction of this claim is not particularly important, except to reassure you that I have not gone too far. (And it only works for this purpose if you

the meaning of $\forall$, since D applies to all vocabulary come what may. So there is no particular reason to prefer this version of the rule. (Another candidate, inspired by Zardini (2011), is considered in Uncut, but ultimately it doesn't work either.)

${ }^{10}$ Motivationally, this is of a piece with the rule D: if something is out of bounds, then filling in more information leaves it out of bounds. But here the extra information comes from substituting terms for variables, rather than adding additional assertions and denials.

${ }^{11}$ Uncut's fuller version I call CL; it's sound and complete for first-order classical logic with equality. 
don't think classical logic itself goes too far!) But the 'only if' direction gives a sense in which Uncut holds that classical logic is 'correct': if classical logic can show that $\Gamma \vdash \Delta$, then it is out of bounds to assert everything in $\Gamma$ while denying everything in $\Delta .{ }^{12}$

\section{Checking in with cut}

Since CL' derives a position iff that position is a classically valid argument, it follows that cut is admissible in CL'. (Classical validity is surely closed under cut, and CL' simply determines classical validity, so what CL' determines is closed under cut.) Cut is not, however, derivable in CL'.

Recall the earlier discussion of admissible and derivable rules: cut's admissibility in CL' does not guarantee that it will remain admissible when additional rules are added. Note what's happened to Swap. While it was admissible in BC, it is not admissible in CL'; the addition of the extra rules governing the UFO vocabulary has changed things. For example, $[p \wedge q \Rightarrow p]$ is derivable in CL', but $[p \Rightarrow p \wedge q]$ is not. Since Swap was merely admissible in BC, this is the kind of thing that can happen.

Since cut too was merely admissible in BC, the same thing might have happened to cut in CL'. But it did not. Still, cut remains merely admissible in CL'. So it is possible that, as we add further rules to capture more of how the bounds behave, cut will go the way of Swap. In CL', nothing breaks cut, but still nothing prevents it being broken; it merely happens to work.

\section{Vague predicates}

When it comes to giving a theory of the meaning of vague predicates, I won't try to give anything like a full theory of the meaning of any particular predicate. Rather, I'll attempt to capture the sense in which vague predicates are tolerant, or insensitive to small-enough differences. Tolerance is the place to focus for two reasons. First, it is what vague predicates have in common, what makes them vague, so we can get something like a general theory of vague predicates by focusing here. Second, it is what gives rise to the paradoxical behaviour of vague

${ }^{12}$ While there are lots of different things someone might mean by calling a logic 'correct', this one isn't totally out of left field. Compare Restall (2005) or the notion MacFarlane (2004) calls Wo-. 
predicates. So if we can capture the sense in which vague predicates are tolerant without problematic conclusions following, we have done enough.

Tolerance for a vague predicate $P$ says that if two things are similar enough in the right ways, then if one of them is $P$ so too is the other. What 'similar enough in the right ways' amounts to differs wildly from predicate to predicate, as well as from context to context; this variation is no part of what Uncut considers. So I will simply assume that, for each predicate $P$, there is a binary relation $\sim_{P}$ expressing this notion. ${ }^{13}$ Moreover, I'll assume that $\sim_{P}$ genuinely is a similarity predicate: that is, that it is reflexive and symmetric, in the sense that the bounds obey the following rules:

$$
\sim \text {-ref-drop: } \frac{\left[t \sim_{P} t, \Gamma \succ \Delta\right]}{[\Gamma \succ \Delta]} \quad \sim \text {-sym-L: } \frac{\left[t \sim_{P} u, \Gamma \succ \Delta\right]}{\left[u \sim_{P} t, \Gamma \succ \Delta\right]} \quad \sim \text { sym-L: } \quad \frac{\left[\Gamma \succ \Delta, t \sim_{P} u\right]}{\left[\Gamma \succ \Delta, u \sim_{P} t\right]}
$$

- -ref-drop says that if a position asserting $t \sim_{P} t$ is out of bounds, this can't be because it asserts $t \sim_{P} t$, it must be out of bounds even without this assertion. This is a way to hold $t \sim_{P} t$ completely innocent. ${ }^{14}$ The two $\sim$ sym rules simply say that asserting or denying a similarity claim amounts to the same as asserting or denying (respectively) its flipped-around version.

Tolerance itself is captured in the following rule:

$$
\text { Tol: } \frac{\left[\Gamma \succ \Delta, t \sim_{P} u\right]}{[P t, \Gamma \succ \Delta, P u]}
$$

Read top to bottom, this says that when $t \sim_{P} u$ is undeniable, then it's out of bounds to assert $P t$ and deny $P u$. To assert $P t$ and deny $P u$, after all, would require denying (at least implicitly) that $t$ and $u$ are similar enough to trigger a tolerance requirement.

This rule on its own involves none of the UFO vocabulary: tolerance can be seen as a constraint relating $P$ to $\sim_{P}$ directly. This is as it should be: while statements of tolerance in sentence form require bits

${ }^{13}$ For relations $R$ with arity $n$, we need $\sim_{R}$ with arity $2 n$, to compare two $n$ tuples for sufficient similarity. Uncut works in this general way, but here I'll stick to unary predicates for simplicity.

${ }^{14}$ Note that $\left[\Rightarrow t \sim_{P} t\right]$ follows from Id via this rule. But the rule is stronger, in the absence of derivable cut, than simply adding the instances of $\left[\Rightarrow t \sim_{P} t\right]$ as initial sequents. See Negri and von Plato (1998) for more. 
of UFO vocabulary, in the end attempts to 'salvage' tolerance by fiddling with the UFO vocabulary end up making tolerance mean something other than what it should. ${ }^{15}$ Tolerance, at its heart, doesn't really involve UFO vocabulary.

Such vocabulary is useful at best in making it explicit in sentence form. And by adding the Tol rule to CL', it becomes possible to see how this explicitation can work. ${ }^{16}$ Consider the following derivation: ${ }^{17}$

$$
\begin{aligned}
& \text { Tol: } \frac{\left[x \sim_{P} y \succ x \sim_{P} y\right]}{\left[P x, x \sim_{P} y \succ P y\right]} \\
& \text { วR: } \\
& \text { วR: } \quad\left[x \sim_{P} y \succ P x \supset P y\right] \\
& \begin{array}{ll}
\forall \mathrm{R}: & \frac{\left[\succ x \sim_{P} y \supset(P x \supset P y)\right]}{\left[\succ \forall y\left(x \sim_{P} y \supset(P x \supset P y)\right)\right]} \\
\forall \mathrm{R}: & \frac{\left[\succ \forall x \forall y\left(x \sim_{P} y \supset(P x \supset P y)\right)\right]}{\left[\Gamma \succ \Delta, \forall x \forall y\left(x \sim_{P} y \supset(P x \supset P y)\right)\right]}
\end{array}
\end{aligned}
$$

Given tolerance, together with the going theory of the UFO vocabulary and the constraints on the bounds encoded in BC, any position denying $\forall x \forall y\left(x \sim_{P} y \supset(P x \supset P y)\right)$ is thus out of bounds. This is not tolerance itself, but a reflection of it visible via the UFO vocabulary, given the rules in play. Tolerance remains as expressed in Tol: a direct connection between $P$ and $\sim_{P}$.

\section{Checking in with cut}

Let CLV' be plus the rules and the Tol rule. This tells us more about the bounds than CL' alone; it now encodes tolerance for vague predicates as well. So a natural question arises: what happens with the sorites paradox?

Let's set one up and see. Consider a sorites series $a, b, c, \ldots, y, z$ of objects, each $P$-similar to its neighbors, but such that $a$ is $P$ and $z$

${ }^{15}$ For more on this line of argument, see also Cobreros et al. $(2012,2017)$.

${ }^{16}$ This is strongly related to the sense of 'making explicit' familiar from Brandom (1994), but in a slightly different theoretical setting. For an attempt to reconcile these settings, see Restall (2008). Fwiw, I think Restall underestimates the differences between his approach and Brandom's; but here isn't the place.

${ }^{17}$ This derivation uses a rule $\supset \mathrm{R}$ that I haven't specified directly here. It's derivable from what I've given, though, if $\supset$ is understood as defined from $\neg$ and $\wedge$ in the usual way. (Uncut specifies a meaning for $\supset$ directly.) 
is not. Let $\Sigma$ be the set of similarity claims $\left\{a \sim_{p} b, b \sim_{p} c, \ldots, y \sim_{p} z\right\}$. Now, consider the following non-derivation:

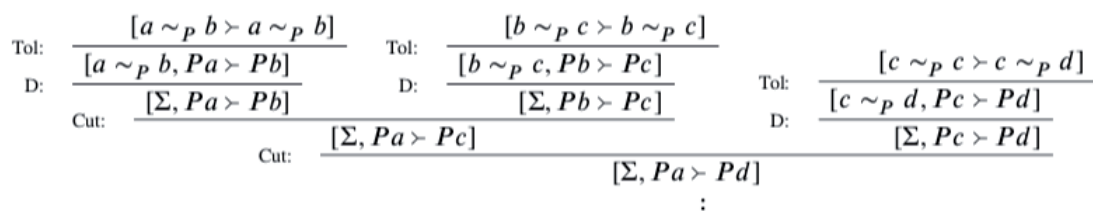

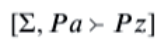

This is a non-derivation because it features repeated use of cut, which is no rule of CLV'. But the only other moves it involves are Id, D, and Tol. So if cut is admissible in CLV', then the position $[\Sigma, P a \Rightarrow P z]$ is derivable in CLV', and so must be out of bounds if CLV' is correct. But this position had better not be out of bounds! It simply captures the soritical setup: all these things are similar to their neighbors, and $a$ is $P$, and $z$ is not. If CLV' declares [ $[, P a \Rightarrow P z]$ to be out of bounds, it has fallen victim to the sorites paradox. So it had better not do this. But then it had better not admit cut.

Fortunately, it does not. To see this, it suffices to show that $[\Sigma, P a \Rightarrow P z]$ is underivable; and to see this, the easiest way is to use a bit of model theory. Before I sketch the idea, though, it's important to remark on just how philosophically unimportant these models are. They certainly are not involved in any way in the going theory of meaning. I am simply using the models as a convenient way to prove that a certain position isn't derivable in CLV'. And CLV' isn't even itself all that philosophically important; it is, I think, correct in its claims about the bounds, but it certainly does not exhaust the truth about them. Moreover, there is nothing particularly important about stopping exactly where CLV' happens to: no special status attaches to those positions whose out-of-boundsness can be established in CLV', as opposed to other outof-bounds positions that are out of bounds for some other reason. So it would be a mistake to interpret models for CLV' as telling us much at all about meaning; they are a mere technical auxiliary to a partial theory of what really matters.

The needed models are quite simple: three-valued models on the so-called strong Kleene scheme, with a few additional constraints on atomic valuations. These models assign to each sentence a value from the set $\{0,1 / 2,1\}$; I won't here rehearse the recursive clauses for complex sentences, but they are straightforward and can be found, for example, in Priest (2008) and Beall and Fraassen (2003). The needed extra 
constraints are: 1) that $t \sim_{P} t$ always takes value 1;2) that $t \sim_{P} u$ and $u \sim_{p} t$ always take the same value as each other; and 3 ) that whenever a valuation assigns $t \sim_{P} u$ a value greater than 0 , then the values it assigns to $P t$ and $P u$ cannot differ by 1 .

Three-valued models are reasonably familiar in the study of nonclassical logics, but less so in the study of classical logics. (Not unknown, though: see for example Girard (1987, Ch. 3), which works with three-valued 'Schütte valuations'.) The key is in the notion of countermodel: one of these three-valued models is a countermodel to a position $[\Gamma \Rightarrow \Delta]$ iff it assigns 1 to everything in $\Gamma$ and 0 to everything in $\Delta$. With this notion of countermodel in mind, it is not hard to show that CLV' is sound: no position derivable in CLV' has a countermodel. The three-valued models alone take care of CL'; the additional restrictions on atomics are what we need for the additional rules present in CLV'.

But now to show that $[\Sigma, P a \Rightarrow P z]$ is not derivable in CLV', it suffices to produce a countermodel to this position; since CLV' is sound, it does not derive anything that has a countermodel. ${ }^{18}$ Such countermodels are easy to come by. Since every sentence in $\Sigma$ must have value 1 on any such countermodel, it follows that no adjacent claims in $P a, P b, \ldots, P z$ can differ in value by 1 . But still $P a$ can have value 1 and $P z$ value 0 , as is needed; while none of the 25 steps along the way can leap from 1 to 0 or 0 to 1 , none needs to; there is always the value $1 / 2$ to be taken along the way.

To bring the discussion of CLV' to a close, then: we have seen that $[\Sigma, P a \Rightarrow P z]$ would be derivable if cut were admissible in CLV'; and we have seen that it is not derivable. So we can conclude that cut is not admissible in CLV'. Moreover, it could not be correctly added: $[\Sigma, P a \Rightarrow P z]$ shouldn't be reckoned out of bounds. Cut, in CLV', goes the way that Swap went in CL'. While these rules may be merely admissible in weaker calculi, once we add to our theory of the bounds we can find counterexamples to them.

Truth

Another important predicate for paradoxes is the truth predicate $T$, and its interactions with names for sentences. I suppose a few things about our language here. First, there is a 'means that' nective $M$; this

${ }^{18}$ It doesn't matter whether CLV' is also complete for these models, because the consequence relation determined by these models is of no import. Soundness suffices in this proof of underivability, and this proof is the only reason we're talking about models at all. 
forms a sentence from a term and a sentence, allowing us to have $t M A$ saying that $t$ means that $A$. With this in hand, we have two double-line truth rules:

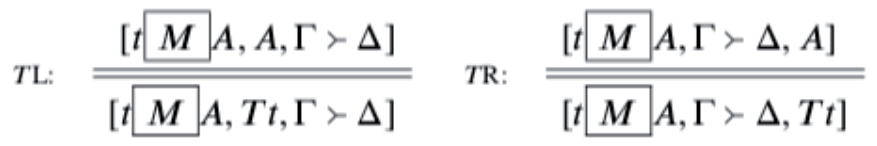

Once a position asserts that $t$ means that $A$, then any constraints that position is subject to around asserting or denying $A$ transfer to the claim that $t$ is true; and any contraints that position is subject to around asserting or denying that $t$ is true transfer to $A$.

With these rules in hand, we can derive something like Tarski's $T$-scheme: ${ }^{19}$

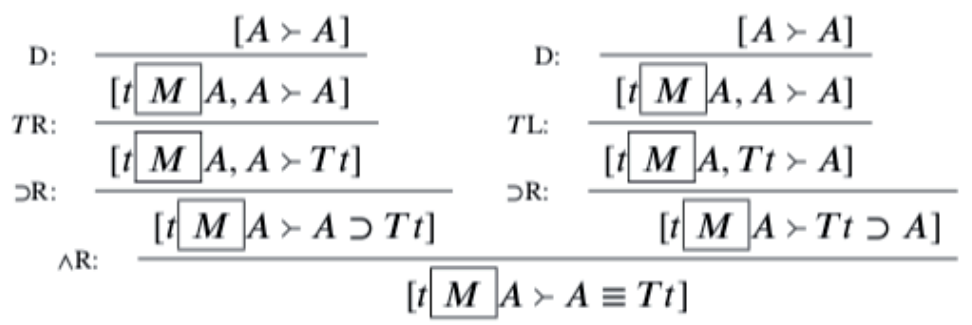

That is, it is out of bounds to assert that $t$ means that $A$ while denying $T t \equiv A$.

In much of the literature on semantic paradoxes, each sentence $A$ is given a canonical name $\langle A\rangle$. If such canonical names are present in the language here (and why not?), then they can be captured with the following rule:

$$
\langle\rangle \text {-drop: } \frac{[\langle A\rangle, M A, \Gamma \succ \Delta]}{[\Gamma \succ \Delta]}
$$

Like the -ref-drop rule in CLV', this rule ensures that asserting that $\langle A\rangle$ means that $A$ is always held totally innocent by the bounds: if a position asserting $\langle A\rangle, M A$ is out of bounds, it can't be because it makes that assertion. It would've been out of bounds anyhow.

19 三 is treated as an abbreviation even in Uncut, but could be treated directly if you like. 
With this rule in hand, it becomes possible to derive the following rules, which might be more familiar. ${ }^{20}$

$$
T\langle\rangle \mathrm{L}: \quad \frac{[A, \Gamma \succ \Delta]}{[T\langle A\rangle, \Gamma \succ \Delta]} \quad T\langle\rangle \mathrm{R}: \quad \frac{[\Gamma \succ \Delta, A]}{\overline{[\Gamma \succ \Delta, T\langle A\rangle]}}
$$

That much, then, gives us a pretty straightforward, naive theory of truth. The claim that $A$ is true, at least when made with $A$ 's canonical name, does not differ at all boundswise from $A$ itself. Each is assertible iff the other is and deniable iff the other is.

This setup, though, allows for a wide range of paradoxes to be formulated. Without some extra story about canonical names, for example, there is no reason we can't have a sentence that contains its own canonical name. For example, we might have a sentence $\neg T t$, where $t$ is the canonical name for $\neg T t$ itself. Let $\lambda$ be such a sentence; that is, $\lambda$ is $\neg T\langle\lambda\rangle$. This is a liar paradox: a sentence that says of itself that it is not true. Similarly, we might have a sentence $T u \supset A$, where $u$ is the canonical name for $T u \supset A$ itself. Let $\kappa_{A}$ be such a sentence; that is, $\kappa_{A}$ is $T\left\langle\kappa_{A}\right\rangle \supset A$. This is a curry paradox (with consequent $A$ ): a sentence that says of itself that if it is true, then $A$. (This is not to mention paradoxical pairs, triples, or other loops, or 'contingent' paradoxes that can be formed from terms other than canonical names by using the $M$ nective, but I'll leave those things for the book.)

\section{Checking in on cut}

Let CLT' be CL' plus the $T$ rules and \langle\rangle -drop. ${ }^{21}$ To see what happens with paradoxical sentences, consider the following nonderivation.

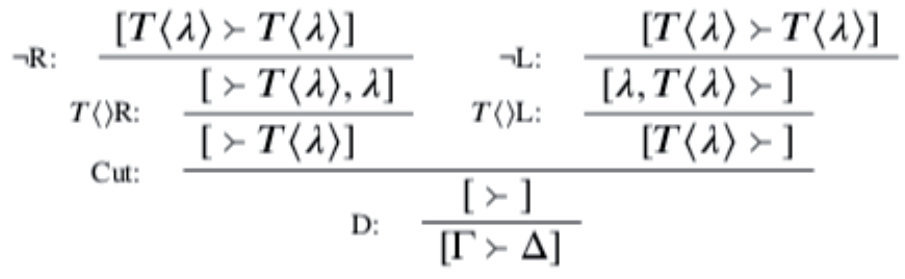

${ }^{20}$ The derivation involves D as well: $\langle A\rangle, M A$ is diluted in to allow use of $T$ rules, then removed by \langle\rangle -drop. The $T\langle\rangle$ rules are the rules I took as governing the truth predicate directly in Ripley (2013b); I think it provides more insight to derive them as here, though.

${ }^{21}$ It's possible to combine the vagueness rules in as well; but let's do it the simple way. 
This is a non-derivation only because of the step of cut; everything else checks out in CLT'. ${ }^{22}$

So if cut is admissible in CLT', then $[\Gamma \Rightarrow \Delta]$ is derivable in CLT'. But this would be a bad result: that's an arbitrary position! CLT', on its intended interpretation, would be saying that every position is out of bounds. It had better not do this; so it had better not admit cut.

Similarly, recalling that $\kappa_{A}$ is the sentence $T\left\langle\kappa_{A}\right\rangle \supset A$, we have the following non-derivation to consider:

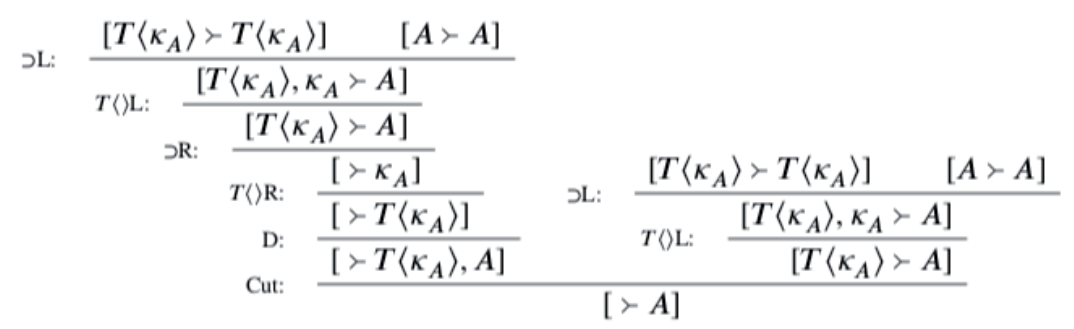

Again, this is a non-derivation only because of the cut. So if cut is admissible in CLT', then $[\Rightarrow A]$ is out of bounds. But this is bad: $A$ here is arbitrary! CLT', on its intended interpretation, would be saying that no sentence is in-bounds deniable. It had better not do this; so again, it had better not admit cut.

To see that all is well, that CLT' indeed doesn't admit cut, we can use the same strategy as for CLV': going through models. Again, these models themselves don't matter at all for the main point; they are just a technical auxiliary to show that certain positions are not derivable in CLT'. The needed kind of model-theoretic strategy is a familiar one in theories of truth. It's based on fixed points of a certain kind of function, and can be found (among other places) in Skolem (1960, 1963); Brady (1971); Martin and Woodruff (1976); Kripke (1975); Leitgeb (1999).

Again, we work with strong Kleene models, now subject to much more complex restrictions on the assignments of values to atomics (and in particular atomics involving the predicate $T$ ). The fixed point strategy mentioned above is what ensures the existence of such models. I won't here go through the details, but if you are familiar with the idea you will

\footnotetext{
${ }^{22}$ If the results of the $\neg$ steps look off to you, remember that $\lambda$ is literally the very sentence $\neg T\langle\lambda\rangle$; there is no need for any rule to connect them/it. If this footnote looks off to you, remember that $\langle A\rangle$ is just a canonical name for $A$; $A$ itself need not 'occur' in any sense in $\langle A\rangle$. (Although of course ' $A$ ' occurs in ' $\langle A\rangle$ '!) If this all seems like gibberish all of a sudden-and really even if it doesn't-I strongly recommend checking in with Meyer and Sylvan (1977, secs. 9-11).
} 
likely be able to see that there are going to be counterexamples available all over the place. In particular, if $\Gamma \cup \Delta$ contains no $T \mathrm{~s},\langle\rangle \mathrm{s}$, or $M / \mathrm{s}$, then $[\Gamma \Rightarrow \Delta]$ is derivable in CLT' iff it is derivable in CL' - that is, iff it is valid in first-order classical logic. This is perhaps a kind of conservativity, although here we have only a single language, so it is not the usual sort.

But many naive theories of truth can achieve that much: total classicality for the non-semantic language. ${ }^{23}$ What CLT' achieves is more: total classicality for the full language. Remember, CL' is in force for the entire language, and it already assures the derivability of every classically valid position. The added rules in CLT' give us a naive truth theory, but they take nothing back. Negation functions just as it does, even when applied to sentences involving semantic vocabulary, or even the paradoxes themselves. So too does the rest of the UFO vocabulary. The conservativeness-like fact is important because it shows us that CLT' has not fallen victim to the paradoxes. It should not be taken to exhaust the senses in which CLT' is classically-minded, though. More important is what was emphasized above: every classically valid position, no matter what vocabulary it uses, is already declared out of bounds by CL', and so by any of our stronger systems.

\section{Validity and the bounds}

Nowadays, paradoxes of validity are back in style. See, for example, Barrio, Rosenblatt, and Tajer (2016); Beall and Murzi (2013); Mates (1965); Read (1979, 2001); Priest (2010); Priest and Routley (1982); Shapiro (2011); Zardini $(2013,2014) .{ }^{24}$ And that is reason enough to discuss them in Uncut: if the tools here can shed light on topics of interest to many, it's worth seeing how. But there is another more pressing reason to discuss the paradoxes of validity here: the risk of revenge. I mean here the kind of revenge where key theoretical terms used to address the paradoxes turn out themselves to be paradox-prone, and to be paradox-prone in such a way that the theory they are part of can't address the new paradoxes it engenders.

There is no risk of revenge of this sort arising here based on 'true'. This is because there is no need, in presenting the theory I've given, ever to use the word 'true' at all. It's an important piece of vocabulary in the

${ }^{23}$ See, for example, Field (2008), Beall (2009), both of which make much of this.

${ }^{24}$ Much of this work considers paradoxes from the medieval logician now known as Pseduo-Scotus. Some credit Bocheński with bringing these paradoxes to the attention of modern logicians, in Bocheński $(1938,1937)$; neither of these seems to be available in English. 
object language, to be sure. After all, it's a necessary ingredient in many of the paradoxes I've been talking about. But there's no need to deploy 'true' in the theory of these paradoxes, or in our theory of meaning at all. As far as I can see, everything I want to say can be straightforwardly said in the 'true'-free fragment of English.

Rather, if there is a risk of revenge, it is in the notion of a position's being out of bounds. Familiar paradoxes of validity can easily be transposed into the language of the bounds. But I can't seriously pretend that I could give a 'bounds'-free version of what I'm doing! The bounds really are the central theoretical notion I'm drawing on. If this notion itself gives rise to paradoxes (as it may well), then those paradoxes too had better be resolvable along the lines I've already presented. It would be no good to say, for example, that all the paradoxes except for 'bounds' paradoxes are to be addressed by thinking carefully about the bounds, while 'bounds' paradoxes are to be addressed by appeal to a Tarskian hierarchy.

Here there is a bit of a puzzle, though. For 'true', there is a reasonably obvious naive theory of it. But what about 'out of bounds'? This is a theoretical notion at its heart; while I intend it to be recognizable from our everyday conversational practice, I have quite deliberately refrained from even attempting a full theory of it. This doesn't get me off the hook, though; it may be that we can say enough about how the notion behaves to see paradoxes in it.

Here's a start. Let $O$ be a predicate in our language that means 'out of bounds', and suppose we have a canonical name $\langle[\Gamma \Rightarrow \Delta]\rangle$ for each position $[\Gamma \Rightarrow \Delta]$. Then we might imagine rules like the following:

$$
O\langle\rangle \mathrm{D}: \quad \frac{\left[\Gamma^{\prime}, \Gamma \succ \Delta, \Delta^{\prime}\right]}{[O\langle[\Gamma \succ \Delta]\rangle, \Gamma \succ \Delta]} \quad O\langle\rangle \mathrm{R}: \quad \frac{\left.\Gamma^{\prime} \succ O\langle[\Gamma \succ \Delta]\rangle, \Delta^{\prime}\right]}{1}
$$

$O\langle\rangle \mathrm{D}$ is named for the rule VD so named in Beall and Murzi (2013) and also discussed under that name in Shapiro (2013). On the present interpretation, $O\langle\rangle \mathrm{D}$ says that if someone has adopted a position $[\Gamma \Rightarrow \Delta]$, then adding to that position an assertion that it is out of bounds results in a position that really is out of bounds. $O\langle\rangle \mathrm{R}$ should be read as subject to a side condition: that $\Gamma^{\prime}$ and $\Delta^{\prime}$ themselves consist entirely of claims about the bounds. Then it is best read contrapositively: it says that if it is in bounds to deny that $[\Gamma \Rightarrow \Delta]$ is out of bounds, then it is in bounds to go on and assert everything in $\Gamma$ and deny everything in $\Delta$. Because of the side condition, it doesn't say that this applies to all background positions (which would be totally implausible); it applies 
only to background positions that themselves make no claims other than bounds claims. It's an S4-ish kind of principle.

Both of these can be questioned; and they are in Uncut. But what I want to point out here is what the situation is if they do apply. So suppose they do. Then paradoxes arise involving the bounds. For example, consider the sentence that says of itself that asserting it is out of bounds. This is a sentence $v$ that is $O\langle[\nu \Rightarrow]\rangle$. This sentence poses a risk of paradox, via the following non-derivation:

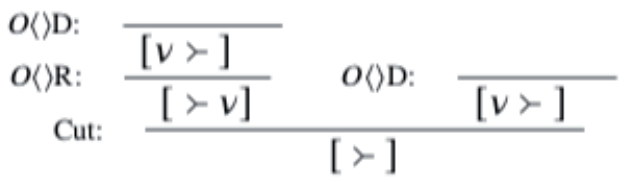

It can be a bit tricky to see, but the only bad step in the above is the application of cut; the uses of the bounds rules are in fact correct.

I hope it's clear how I'm going to respond to this paradox. It's just like the other paradoxes we've looked at: the step of cut cannot be justified, and so this non-derivation doesn't in fact go through. The empty position cannot be derived even if we take up constraints like $O\langle\rangle \mathrm{D}$ and $O\langle\rangle$ R. This underivability claim, like the others, can be shown by a detour through models; details are in the book. Paradoxes involving the bounds can be handled in just the same way as more familiar paradoxes; the present approach doesn't need to use any ad hoc machinery to address them. Revenge is thus avoided.

\section{Summing up}

It started out seeming like the paradoxes posed a threat to things meaning what they seem to. But the threat has simply failed to materialize. The key is in the bounds-based theory of meaning, which helps us to see an important sense in which all the desired principles governing the involved vocabulary can hold without trouble. In seeing that no trouble arises, though, it is central to allow for cut to fail for the bounds.

\section{References}

Barrio, E., Rosenblatt, L., \& Tajer; D. (2016). Capturing naive validity in the cut-free approach. Synthese. https://doi.org/10.1007/s11229016-1199-5

Beall, J. (2009). Spandrels of truth. Oxford University Press. 
Beall, J., \& Murzi, J. (2013). Two flavors of curry's paradox. Journal of Philosophy, 110(3), 143-165.

Beall, J., \& van Fraassen, B. C. (2003). Possibilities and paradox: An introduction to modal and many-valued logic. Oxford University Press.

Bocheński, J. M. (1937). Notes historiques sur les propositions modales. Revue Des Sciences Philosophiques et Théologiques, 26, 673-692.

Bocheński, J. M. (1938). De Consequentiis Scholasticorum Earumque Origine. Angelicum, 15, 92-109.

Brady, R. T. (1971). The consistency of the axioms of abstraction and extensionality in a three-valued logic. Notre Dame Journal of Formal Logic, 12(4), 447-453.

Brandom, R. (1994). Making it explicit. Harvard University Press.

Cobreros, P., Égré, P., Ripley, D., \& van Rooij, R. (2012). Tolerant, Classical, Strict. Journal of Philosophical Logic, 41(2), 347-385.

Cobreros, P., Égré, P., Ripley, D., \& van Rooij, R. (2017). Tolerant reasoning: Nontransitive or Nonmonotonic? Synthese. https://doi. org/10.1007/s11229-017-1584-8

Field, H. (2008). Saving truth from paradox. Oxford University Press.

Girard, J.-Y. (1987). Proof theory and logical complexity (vol. I). Bibliopolis.

Kripke, S. (1975). Outline of a theory of truth. Journal of Philosophy, 72(19), 690-716.

Leitgeb, H. (1999). Truth and the liar in De Morgan-valued models. Notre Dame Journal of Formal Logic, 40(4), 496-514.

MacFarlane, J. (2004). In what sense (if any) is logic normative for thought? https://johnmacfarlane.net/normativity_of_logic.pdf

Martin, R. L., \& Woodruff, P. W. (1976). On representing 'true-in-L' in L. In A. Kasher (Ed.), Language in Focus: Foundations, Methods and Systems (pp. 113-117). Reidel.

Mates, B. (1965). Pseudo-Scotus on the soundness of consequentiae. In A.-T. Tymieniecka (Ed.), Contributions to logic and methodology in honor of J. M. Bocheński (pp. 132-141). North-Holland.

Meyer, R., \& Sylvan, R. (1977). Extensional Reduction II. Manuscript. Partially published in R. Brady (Ed.) (2003), Relevant logics and their rivals, vol. 2. Ashgate Publishing.

Negri, S., \& von Plato, J. (1998). Cut elimination in the presence of axioms. The Bulletin of Symbolic Logic, 4(4), 418-435.

Price, H. (1990). Why 'Not’? Mind, 99(394), 221-238.

Priest, G. (2008). An introduction to non-classical logic: From if to is ( $2^{\text {nd }}$ ed). Cambridge University Press. 
Priest, G. (2010). Hopes fade for saving truth. Philosophy, 85(1), 109-140.

Priest, G., \& Routley, R. (1982). Lessons from Pseudo-Scotus.Philosophical Studies, 42(2), 189-199.

Read, S. (1979). Self-reference and validity. Synthese, 42(2), 265-274.

Read, S. (2001). Self-reference and validity revisited. In M. Yrjönsuuri (Ed.), Medieval Formal Logic (183-196). Springer.

Restall, G. (2005). Multiple conclusions. In P. Hájek, L. Valdés-Villanueva \& D. Westerståhl (Eds.), Logic, methodology, and philosophy of science: Proceedings of the Twelfth International Congress (pp. 189-205). Kings' College Publications.

Restall, G. (2008). Assertion, denial, commitment, entitlement, and incompatibility (and some consequence). Studies in Logic, 1(1), 26-36.

Restall, G. (2009). Truth values and proof theory. Studia Logica, 92(2), 241-264.

Restall, G. (2013). Assertion, denial, and non-classical theories. In K. Tanaka, F. Berto, E. Mares \& F. Paoli (Eds.), Paraconsistency: Logic and applications (pp. 81-100). Springer.

Ripley, D. (2013a). Paradoxes and failures of cut. Australasian Journal of Philosophy, 91(1), 139-164.

Ripley, D. (2013b). Revising up: Strengthening classical logic in the face of paradox. Philosophers' Imprint, 13(5), 1-13.

Ripley, D. (2015a). Anything Goes. Topoi, 34(1), 25-36.

Ripley, D. (2015b). Comparing substructural theories of truth. Ergo, 2(13), 299-328.

Ripley, D. (2017a). Bilateralism, coherence, warrant. In F. Moltmann \& M. Textor (Eds.), Act-based conceptions of propositional content: Contemporary and historical perspectives (pp. 307-324). Oxford University Press.

Ripley, D. (2017b). On the 'transitivity' of consequence relations. Journal of Logic and Computation, 28(2), 433-450.

Shapiro, L. (2011). Deflating logical consequence. Philosophical Quarterly, 61(243), 320-342.

Shapiro, L. (2013). Validity curry strengthened. Thought, 2(2), 100-107.

Shapiro, L. (2016). The very idea of a substructural approach to paradox. Synthese, 1-20. https://doi.org/10.1007/s11229-016-1230-x.

Skolem, T. (1960). A set theory based on a certain three-valued logic. Mathematica Scandinavica, 8(1), 127-136.

Skolem, T. (1963). Studies on the axiom of comprehension. Notre Dame Journal of Formal Logic, 4(3), 162-170.

Zardini, E. (2011). Truth without contra(di)ction. Review of Symbolic 
Logic, 4(4), 498-535.

Zardini, E. (2013). Naive logical properties and structural properties. Journal of Philosophy, 110(11), 633-644.

Zardini, E. (2014). Naive truth and naive logical properties. Review of Symbolic Logic, 7(2), 351-384.

Received $26^{\text {th }}$ January 2018; accepted $1^{\text {st }}$ February 2018. 